\title{
Hereditary polyhedra with planar regular faces*
}

\author{
Egon Schulte ${ }^{\dagger}$ \\ Department of Mathematics, Northeastern University, \\ Boston, MA 02115, USA \\ Asia Ivić Weiss $\ddagger$ \\ Department of Mathematics and Statistics, York University, \\ Toronto, Ontario M3J 1P3, Canada \\ In memory of Norman Johnson, our friend and colleague.
}

Received 18 December 2018, accepted 6 July 2019, published online 10 August 2020

\begin{abstract}
A skeletal polyhedron in Euclidean 3-space is called hereditary if the symmetries of each face extend to symmetries of the entire polyhedron. In this paper we describe the finite hereditary skeletal polyhedra which have regular convex polygons or regular starpolygons as faces.
\end{abstract}

Keywords: Symmetries of polyhedra, geometric polyhedral, uniform polyhedra.

Math. Subj. Class. (2020): 51M20, 52B05, 52B22

\section{Introduction}

In the design of polyhedral structures with high symmetry it is quite natural to proceed from a highly symmetric structure of lower rank (or dimension) and ask for the symmetries of the lower rank structure to be preserved for the entire structure. The entire structure then inherits the symmetries of the lower rank structure. For example, the Platonic solids and

\footnotetext{
* Special thanks go to Peter McMullen for pointing out the omission of a known uniform polyhedron from the list in an earlier version of the manuscript. We did know about the polyhedron but a bit of absentmindedness had caused us to forget to include it. His forthcoming paper [13] will describe an alternative approach to the enumeration presented here, and will also deal with the case of skew faces. We would also like to thank Tomaž Pisanski and the anonymous referee for helpful comments which have improved the paper.

${ }^{\dagger}$ Supported by the Simons Foundation Award No. 420718.

${ }^{\ddagger}$ Corresponding author. Supported by NSERC grant.

E-mail addresses: e.schulte@ northeastern.edu (Egon Schulte), weiss@yorku.ca (Asia Ivić Weiss)
} 


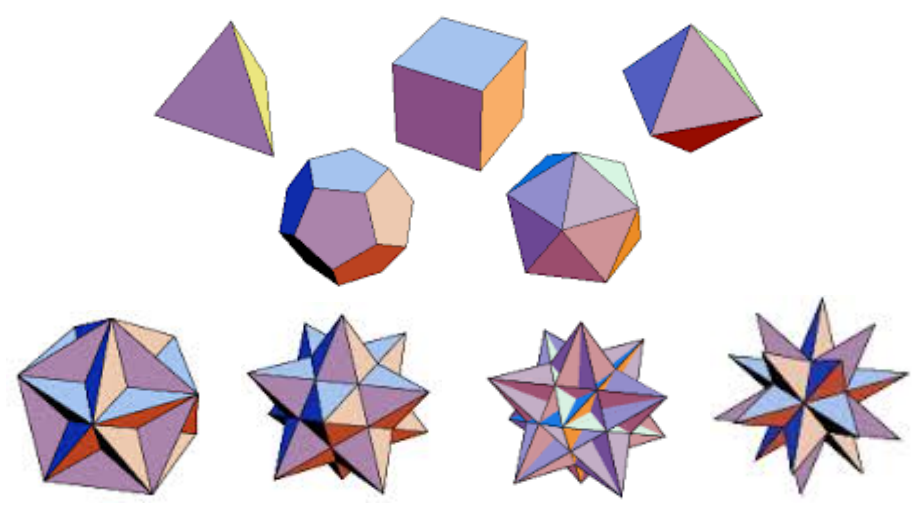

Figure 1: The finite regular polyhedra with planar faces (the five Platonic solids and the four Kepler-Poinsot polyhedra).

the Kepler-Poinsot polyhedra shown in Figure 1 have the property that each symmetry of each face extends to the entire figure (see [1]).

In this paper we study finite hereditary geometric polyhedra in $\mathbb{E}^{3}$ with planar regular faces. Here a polyhedron is viewed as a finite geometric graph with a distinguished class of polygonal cycles, called faces, such that two faces meet at each edge. A polyhedron is hereditary if each symmetry of each face extends to a symmetry of the polyhedron. For instance, all of the eighteen finite regular polyhedra in $\mathbb{E}^{3}$ are hereditary. Recall that these polyhedra consist of the nine classical regular polyhedra, that is, the Platonic solids and the Kepler-Poinsot polyhedra, and their Petrie duals (see [4, 5, 6, 15] or [16, Ch. 7E]). Hereditary polyhedra with regular faces are highly-symmetric polyhedra and have maximal local symmetry (with respect to faces).

For hereditary polyhedra, the regularity assumption on the faces has strong implications for the geometry and enables us to say a great deal about them. Our main result is the following theorem.

Theorem 1.1. The finite hereditary polyhedra with planar regular faces in $\mathbb{E}^{3}$ are

(a) the nine classical regular polyhedra (Platonic solids and Kepler-Poinsot polyhedra),

(b) the medials of the eighteen finite regular polyhedra,

(c) the great ditrigonal icosidodecahedron $(5 \cdot 3)^{3}$,

(d) the small ditrigonal icosidodecahedron $\left(\frac{5}{2} \cdot 3\right)^{3}$, and

(e) the ditrigonal dodecadodecahedron $\left(5 \cdot \frac{5}{2}\right)^{3}$.

Theorem 1.1 might give the false impression that there are $9+18+3=30$ finite hereditary polyhedra with planar regular faces in $\mathbb{E}^{3}$. However, some polyhedra are counted more than once in the theorem, since pairs of dual finite regular polyhedra have the same medials, and the regular octahedron also occurs as the medial of the regular tetrahedron. The exact number of polyhedra turns out to be 25 , not 30 . 

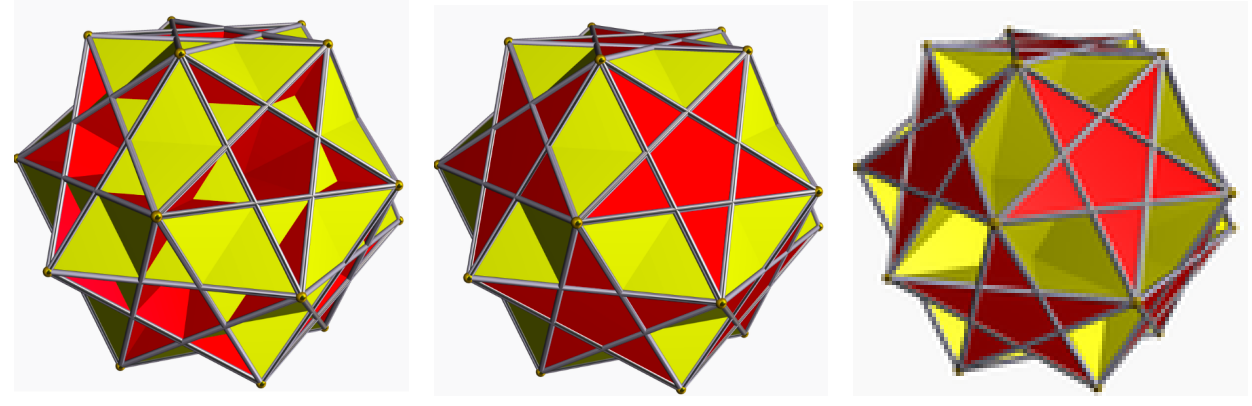

Figure 2: The great ditrigonal icosidodecahedron $(5 \cdot 3)^{3}$ (left), small ditrigonal icosidodecahedron $\left(\frac{5}{2} \cdot 3\right)^{3}$ (middle), and ditrigonal dodecadodecahedron $\left(5 \cdot \frac{5}{2}\right)^{3}$ (right).

Theorem 1.2. Up to similarity, there are precisely 25 finite hereditary polyhedra with planar regular faces in $\mathbb{E}^{3}$.

We list these polyhedra and some of their properties in Table 1 at the end of the paper.

Figure 2 shows the three exceptional polyhedra listed in parts (c), (d), and (e) of Theorem 1.1. The Petrie duals of the classical regular polyhedra are hereditary (in fact, regular) polyhedra but have skew faces and therefore do not occur in the list of Theorem 1.1.

Historically, polyhedra with regular faces have attracted a lot of attention (for example, see [12]). Usually these figures were convex polyhedra or star-polyhedra. This paper is dedicated to the late Norman Johnson who has greatly contributed to our understanding of the geometry, combinatorics, and algebra of polyhedra and more general polyhedral structures (see Johnson $[11,10]$ ).

\section{Basic notions and facts}

A (finite) polygon, or more specifically a $p$-gon (with $p \geqslant 3$ ), consists of a sequence $v_{1}, v_{2}, \ldots, v_{p}$ of $p$ distinct points in $\mathbb{E}^{3}$, as well as of the line segments $\left[v_{i}, v_{i+1}\right]$ for $i=1, \ldots, p$ (with indices considered $\bmod p$ ). The points are the vertices and the line segments are the edges of the polygon. A polygon is planar if its vertices (and edges) lie in a plane; otherwise the polygon is skew, or non-planar.

An incident vertex-edge pair of a polygon $F$ is called a flag (or sometimes an arc) of $F$. A polygon $F$ is said to be regular if its symmetry group $G(F)$ is transitive on the flags of $F$. Recall that the (geometric) symmetry group of a figure is the group of all isometries of the ambient space that leave the figure invariant; its elements are the symmetries of the figure. Thus a planar polygon has a planar symmetry group.

A planar regular polygon with $p$ vertices is necessarily (the graph consisting of the vertices and edges of) a regular convex $p$-gon, denoted $\{p\}$, or a regular star polygon, denoted $\left\{\frac{p}{d}\right\}$, with $(p, d)=1$ (see [1]). Recall that the vertices of $\left\{\frac{p}{d}\right\}$ are the same as those of $\{p\}$, and that its edges successively connect vertices $d$ steps apart on $\{p\}$, beginning at the first vertex (say) of $\{p\}$. The symmetry group of a planar regular $p$-gon, the (planar) dihedral group $D_{p}$ of order $2 p$, by definition consists of 2 -dimensional isometries, and is generated by two reflections (in lines). When the $p$-gon is viewed as lying in a plane of $\mathbb{E}^{3}$, these reflections extend in an obvious way to plane reflections generating a reflection group 
in $\mathbb{E}^{3}$ isomorphic to $D_{p}$. We call this group the trivial extension of $D_{p}$ to $\mathbb{E}^{3}$, and by abuse of terminology and notation we also call this extension the dihedral group $D_{p}$.

A skew regular $p$-gon must have an even number of vertices $p$. Its symmetry group is generated by a plane reflection (interchanging the edges at a vertex) and a half-turn (about the midpoint of an edge containing that vertex), and again is isomorphic to $D_{p}$; note that in this case the product of the two generators is a rotatory reflection (the composition of a rotation, and a reflection in a plane perpendicular to the rotation axis).

A (finite skeletal) polyhedron $P$ in $\mathbb{E}^{3}$ consists of a finite set of distinct points, called vertices, a set of line segments connecting vertices, called edges, and a set of polygons made up of edges, called faces, with the following three properties.

- The graph formed by the vertices and edges of $P$, called the edge graph (or 1skeleton) of $P$, is connected.

- The vertex-figure at each vertex of $P$ is connected. By the vertex-figure of $P$ at a vertex $v$, denoted $P / v$, we mean the graph whose vertices are the neighbors of $v$ in the edge graph of $P$ and whose edges are the line segments $(u, w)$, where $(u, v)$ and $(v, w)$ are edges of a common face of $P$.

- Each edge of $P$ belongs to exactly two faces of $P$.

Note that a polyhedron is a geometric realization in $\mathbb{E}^{3}$, in the sense of [16, Ch. 5], of a finite abstract polyhedron and its respective map on a closed surface.

A skeletal polyhedron $P$ is called planar-faced or skew-faced respectively, if all faces of $P$ are planar or some faces of $P$ are skew. We call a polyhedron $P$ regular-faced if each face of $P$ is a regular polygon (and thus is a polygon with maximum possible symmetry).

The symmetry group of a (finite) polyhedron $P$ is a finite group of isometries of $\mathbb{E}^{3}$ and thus fixes the centroid of the vertex set of $P$, which we call the center of $P$. Throughout we assume that the center of $P$ lies at $o$, the origin of $\mathbb{E}^{3}$. We call a face of $P$ central if the center of $P$ is the centroid of the vertex-set of the face. If a central face is planar then its ambient plane passes through the center of $P$. A non-central face of $P$ is a face of $P$ which is not central.

A polyhedron $P$ is said to be (geometrically) hereditary if the symmetry group $G(F)$ of each face $F$ of $P$ can be viewed as a subgroup of the symmetry group $G(P)$ of $P$, or more informally, if each symmetry of each face $F$ of $P$ extends to a symmetry of $P$. (Note that the abstract polyhedron underlying a geometrically hereditary polyhedron $P$ with regular faces is also combinatorially hereditary, in the sense that the combinatorial automorphism group of each face extends to a subgroup of the automorphism group of $P$. Combinatorially hereditary abstract polyhedra were shown in [17] to be regular or 2-orbit of type $2_{01}$; see also [8] and [9].)

For a face $F$ of a hereditary polyhedron $P$ we let $G_{P}(F)$ denote the subgroup of $G(P)$ consisting of the symmetries of $P$ which extend symmetries of $F$.

If a face $F$ of a hereditary polyhedron $P$ is skew, then each symmetry of $F$ already is 3-dimensional and thus the extended symmetry is the symmetry itself; that is, $G_{P}(F)=$ $G(F)$. Note that a regular skew face of a hereditary polyhedron must necessarily be central, since $o$ and the center of the face must be invariant under the symmetries of the face.

However, this is different for planar faces. If a planar face $F$ of $P$ is non-central, then the extensions of the symmetries of $F$ to symmetries of $P$ still are unique, since $o$ must be invariant; in this case, if $F$ is a regular $p$-gon and $G(F)=D_{p}$ is identified with its trivial extension to $\mathbb{E}^{3}$, then $G_{P}(F)=G(F)=D_{p}$. If a planar face $F$ of $P$ is central, 
however, then the symmetries of $F$ may occur in $P$ in one of two ways; in fact, if $F$ admits a reflection symmetry in a line $l$ (through $o$ ), then this planar symmetry of $F$ may occur either as a reflection in a plane through $l$ perpendicular to the plane of $F$ or as a half-turn about $l$. We later see that, for a regular-faced hereditary polyhedron with planar faces, each symmetry of a central face $F$ has a unique extension to $P$ (so reflective symmetries of $F$ can not extend to both a plane reflection and a half-turn). The geometry of the group $G_{P}(F)$ then depends on the nature of these extensions but is still isomorphic to $G(F)$.

An incident vertex-edge-face triple of a polyhedron $P$ is called a flag of $P$. A polyhedron $P$ is (geometrically) regular if its symmetry group $G(P)$ is transitive on the flags of $P$. Regular polyhedra are hereditary regular-faced polyhedra.

An incident vertex-edge pair of a polyhedron $P$ is called an $\operatorname{arc}$ of $P$. We say that $P$ is vertex-, edge-, or arc-transitive if $G(P)$ acts transitively on the vertices, edges, or arcs of $P$, respectively. Clearly, if $P$ is arc-transitive, then $P$ is vertex-transitive and edge-transitive. For a vertex $v$ of $P$, let $G_{v}(P)$ denote the stabilizer of $v$ in $G(P)$.

Proposition 2.1. Let $P$ be a (finite) hereditary polyhedron with regular faces in $\mathbb{E}^{3}$. Then $P$ is arc-transitive. In particular, the vertex-figures are mutually equivalent under $G(P)$, and the stabilizer $G_{v}(P)$ of a vertex $v$ of $P$ in $G(P)$ acts transitively on the vertices of the vertex-figure $P / v$ at $v$. Moreover, the vertex-figures are planar.

Proof. The first statement follows from [17, Prop. 1]. Any two $\operatorname{arcs}$ of $P$ are related via a finite sequence of arcs such that successive arcs in the sequence are arcs of a common face of $P$. Since the faces are regular and thus arc-transitive under their own symmetry group, and since every symmetry of a face of $P$ extends to a symmetry of $P$, it follows that $P$ is arc-transitive. Thus $P$ is vertex-transitive and the stabilizer $G_{v}(P)$ of a vertex $v$ in $G(P)$ acts transitively on the vertices of the vertex-figure $P / v$ at $v$.

Moreover, the vertex-figures must be planar since $P$ is finite. In fact, by the vertextransitivity, the vertices of $P$ must all lie on a sphere centered at $o$; and since $G_{v}(P)$ acts vertex-transitively on $P / v$, the vertices of $P / v$ must all lie on a sphere centered at $v$. Thus the vertices of $P / v$ all lie on the intersection of the two spheres, which is a circle. This shows that $P / v$ is planar.

It follows that every hereditary polyhedron with regular faces is a uniform polyhedron in $\mathbb{E}^{3}$. Recall that a uniform polyhedron is a vertex-transitive polyhedron with regular faces (see [1]).

The finite uniform polyhedra with planar faces were classified by Coxeter, LonguetHiggins and Miller [2] in 1954. It is customary to describe these polyhedra by a vertexsymbol $\left(n_{1} \cdot n_{2} \cdot \ldots \cdot n_{q}\right)$ with integral or rational entries. Here $q$ is the valency of a vertex, and the entries $n_{1}, \ldots, n_{q}$ represent the faces that surround a vertex, in cyclic order, such that the face corresponding to $n_{i}$ has Schläfli symbol $\left\{n_{i}\right\}$ for $i=1, \ldots, q$ (thus the face is a convex regular $n_{i}$-gon if $n_{i}$ is an integer, or a regular star-polygon $\left\{n_{i}\right\}$ if $n_{i}$ is a fraction). For example, the small ditrigonal icosidodecahedron occurring in Theorem 1.1 and shown in Figure 2 has vertex-symbol $\left(\frac{5}{2} \cdot 3 \cdot \frac{5}{2} \cdot 3 \cdot \frac{5}{2} \cdot 3\right)$, indicating that at each vertex three pentagrams $\left\{\frac{5}{2}\right\}$ and three triangles $\{3\}$ alternate; the symbol is abbreviated to $\left(\frac{5}{2} \cdot 3\right)^{3}$.

For our purposes, we further refine the vertex-symbol to indicate the presence of central faces. If a polyhedron has a central face, then the superscript “*” in its refined vertex-symbol indicates that the corresponding face type represents a central face of the polyhedron. For example, the symbol $\left(\frac{5}{2} \cdot 6^{*} \cdot \frac{5}{2} \cdot 6^{*}\right)$ would represent a polyhedron in which two pentagrams 
$\left\{\frac{5}{2}\right\}$ and two central regular hexagons $\{6\}$ alternate at a vertex. Thus, a polyhedron has a central face if and only if a " $*$ " occurs in its refined vertex-symbol.

No classification of the finite uniform polyhedra with skew faces is known to date, but new uniform polyhedra with skew faces have recently been found in [21, 23, 24]. See also Grünbaum [7].

Note that, for a regular-faced hereditary polyhedron with vertices of valency $q$, the vertex stabilizers $G_{v}(P)$ may not be isomorphic to $D_{q}$, even though $G_{v}(P)$ acts vertextransitively on the $q$-gonal vertex-figure $P / v$ at $v$. However, the following proposition holds.

Proposition 2.2. Let $P$ be a (finite) regular-faced hereditary polyhedron without central faces and with vertices of valency $q$. Then, for every vertex $v$ of $P$, the vertex stabilizer $G_{v}(P)$ of $v$ in $G(P)$ is a dihedral subgroup $D_{q}$, if $q$ is odd, or contains a dihedral group $D_{q / 2}$ which acts transitively on the $q$ vertices of the vertex-figure $P / v$, if $q$ is even. Moreover, if $q$ is odd then $P$ is a regular polyhedron.

Proof. By the vertex-transitivity of $P$ it suffices to consider the vertex stabilizer subgroup for a single vertex. So let $v$ be a vertex of $P$. Since the faces are non-central, each face $F$ of $P$ at $v$ contributes to $G(P)$ a unique plane reflection which leaves both $F$ and $v$ invariant and interchanges the two edges of $F$ meeting at $v$. This holds regardless of whether $F$ is planar or skew. These reflections for the $q$ faces at $v$ generate a dihedral group $D_{q}$ if $q$ is odd, or $D_{q / 2}$ if $q$ is even. Note that this subgroup of $G_{v}(P)$ acts vertex-transitively on $P / v$.

If $q$ is odd, then the dihedral subgroup $D_{q}$ of $G_{v}(P)$ must necessarily coincide with $G_{v}(P)$. Hence $G_{v}(P)$ must contain symmetries that swap adjacent faces of $P$ meeting at $v$. Thus $G_{v}(P)$ must act flag-transitively on the vertex-figure $P / v$ at $v$, and since $P$ is vertextransitive, $G(P)$ itself must act flag-transitively on $P$. Thus $P$ is a regular polyhedron.

Proposition 2.1 is telling us that the vertex-figures of hereditary regular-faced polyhedra must be congruent. The faces, however, need not be congruent (even though all are regular). On the other hand, by the edge-transitivity of $P$ there can be at most two face orbits under $G(P)$. If indeed there are two face orbits, then the two faces of $P$ meeting at an edge of $P$ must lie in different face orbits under $G(P)$, and hence $q$ must be even.

If a hereditary regular-faced polyhedron $P$ has a central planar face, then each face adjacent to any such face must either be a non-central planar face or a skew face, as we explain in a moment. As a consequence, by the edge-transitivity of $P$, each edge of $P$ must lie in a central planar face as well as in a non-central planar face or a skew face. In particular, $G(P)$ must have two face orbits, one consisting of the central planar faces and the other of the non-central planar faces or the skew faces. Further, $q$ must be even.

Note that $P$ cannot have a pair of adjacent central planar faces. In fact, any such pair of faces would necessarily have to lie in the same plane and share $o$ as the center. The edge transitivity then would force the entire polyhedron $P$ to lie in this plane, with all faces sharing the same symmetry group. However this is impossible since then all faces would have to coincide; in fact, since the faces are regular, the symmetry group of a face is entirely determined by the angle subtended at $o$ by one of its edges.

We noted earlier that, for non-central planar faces or skew faces of a hereditary regularfaced polyhedron $P$, there is just one way in which a planar symmetry of a face can extend to a symmetry of $P$. This also remains true for the central planar faces of $P$, for the following reason. Suppose $F$ is a central planar face and $l$ is a reflection line for $F$ in the 
plane that contains $F$. There are only two isometries of $\mathbb{E}^{3}$ which extend the 2-dimensional reflection in $l$, namely the half-turn about $l$ and the reflection in the plane perpendicular at $l$ to the plane of $F$. Now if both isometries are symmetries of $P$, then so is their product, which is the reflection in the plane containing $F$. However, this reflection cannot be a symmetry, since the image of an adjacent (non-central planar, or skew, respectively) face $G$ of $F$ under this reflection would yield another (non-central planar, or skew) face $G^{\prime}$ of $F$ meeting $F$ at the same edge as $G$. This is impossible. Thus each planar reflection symmetry of $F$ extends in just one way to a symmetry of $P$. This forces the same to be true for the rotational symmetries of $F$.

We also require the following two well-known concepts for polyhedra (see $[1,16,19])$.

A Petrie polygon of a regular polyhedron $P$ in $\mathbb{E}^{3}$ is a path along edges of $P$ such that every two, but no three, consecutive edges belong to a face of $P$ (see [1, 3, 19]). Every regular polyhedron $P$ gives rise to a new structure, denoted $P^{\pi}$ and called the Petrie dual, or Petrial, of $P$, which in most cases is again a polyhedron (see [16, Lemma 7B3]). For example, $\{4,3\}^{\pi}$, the Petrial of a cube, is a polyhedron with four hexagonal skew faces.

Given a regular polyhedron $P$ in $\mathbb{E}^{3}$ the medial $\mathrm{Me}(P)$ is a new structure, usually a polyhedron, with faces of two kinds: the polygons with vertices at the midpoints of consecutive edges in a face of $P$, and the polygons with vertices at the midpoints of consecutive edges meeting at a vertex of $P$. The medial of a regular polyhedron may not always be a polyhedron. For example, in the blended polyhedron $\{3,6\} \#\{\}$ edges can cross at midpoints and hence the edge midpoints occupy the same point in $\mathbb{E}^{3}$ (see [16, Ch. 7E]). Thus its medial is not a polyhedron.

\section{Planar-faced polyhedra with no central faces}

In the next two sections, we describe and characterize the finite regular-faced hereditary polyhedra $P$ in $\mathbb{E}^{3}$ all of whose faces are planar. Their vertex-figures are also planar, by Proposition 2.1. Our analysis of these polyhedra greatly depends on whether or not they have central faces.

In this section, we deal with the finite planar-faced hereditary polyhedra $P$ with no central faces. Polyhedra with central faces are discussed in the next section.

So let $P$ be a finite hereditary polyhedron with regular faces all of which are planar and non-central, and with vertices of valency $q$. Recall our standing assumption that the center of $P$ lies at $o$. Then each symmetry of each face $F$ of $P$ is extended to $P$ in the trivial way. Thus the subgroup $G_{P}(F)$ of $G(P)$ is a dihedral group, namely the trivial extension of the dihedral symmetry group $G(F)$ of $F$. In particular, $G(P)$ must contain many plane reflections. It follows that $G(P)$ must be the full symmetry group of a Platonic solid $R$ (say), and that the face centers of $P$, being centers of rotation of a regular face, must lie on axes of rotation of $R$. In particular, each face $F$ of $P$ must have 3,4 or 5 vertices.

As $P$ has no central faces, we know from Proposition 2.2 that the vertex stabilizer $G_{v}(P)$ of a vertex $v$ is a dihedral subgroup $D_{q}$ if $q$ is odd, or contains a dihedral subgroup $D_{q / 2}$ if $q$ is even. In particular, each vertex $v$ of $P$ is a center of rotational symmetry of $P$ about an axis passing through $v$ and $o$. Thus $v$ must lie on a rotation axis of the underlying Platonic solid $R$ and therefore coincide with a vertex, the midpoint of an edge, or the center of a face of $R$, up to rescaling of $R$. Clearly, by replacing $R$ by its dual (if need be), we may assume that the vertices of $P$ lie either at vertices or edge midpoints of $R$. Then, since $G(P)=G(R)$ and $P$ is vertex-transitive, the vertex set of $P$ coincides with either the full 
vertex set of $R$ or the full set of edge midpoints of $R$.

If the vertex valency $q$ is odd, then Proposition 2.2 is telling us that $P$ is a regular polyhedron and that $G_{v}(P)=D_{q}$ for every vertex $v$ of $P$. Thus the vertex-figures are congruent regular polygons, and by Proposition 2.1 are planar. Inspection of the list of finite regular polyhedra in $\mathbb{E}^{3}$ then establishes the following proposition (see [16]).

Proposition 3.1. Let $P$ be a (finite) hereditary polyhedron with planar regular faces, all non-central, and with vertices of odd valency. Then P is either a Platonic solid or a KeplerPoinsot polyhedron.

When the vertex valency $q$ of $P$ is even, the $(q / 2)$-fold rotation about a vertex of $P$ has order $2,3,4$, or 5 , and so $q=4,6,8$, or 10 . This case is more involved. The remainder of this section deals with the proof of the following proposition.

Proposition 3.2. Let $P$ be a (finite) hereditary polyhedron with planar regular faces, all non-central, and with vertices of even valency. Then $P$ is the medial of a Platonic solid, the medial of a Kepler-Poinsot polyhedron, a small ditrigonal icosidodecahedron $\left(\frac{5}{2} \cdot 3\right)^{3}$, a great ditrigonal icosidodecahedron $(5 \cdot 3)^{3}$, or a ditrigonal dodecadodecahedron $\left(5 \cdot \frac{5}{2}\right)^{3}$.

Proof. The proof of Proposition 3.2 investigates the two possible placements of the vertices of $P$ on $R$, with $R$ as above, namely either at the vertices of $R$ (Case 1) or at the edge midpoints of $R$ (Case 2). So let the vertex valency $q$ of $P$ be even.

Case 1: The vertices of $P$ lie at the vertices of $R$.

We first rule out the possibility that $R$ is a tetrahedron $\{3,3\}$, a cube $\{4,3\}$, or an icosahedron $\{3,5\}$. Clearly, since $q \geqslant 4, R$ cannot be $\{3,3\}$. To see that $R=\{3,5\}$ is impossible, we note that since $q$ is even and $P$ in this case has 5-fold rotational symmetries about its vertices, $q$ must be 10 and the vertex-figures of $P$ must be planar decagons; however, no ten vertices of $\{3,5\}$ lie in a common plane. Similarly, $R=\{4,3\}$ is impossible since no six vertices of $\{4,3\}$ lie in a common plane.

If $R=\{3,4\}$, then clearly $q=4$ and the neighbors of a vertex $v$ in $P$ are just those in $R$. Since a 4-fold rotation about $v$ must cyclically permute the faces of $P$ at $v$, and since the faces are planar, the faces of $P$ at $v$ must necessarily be the faces of $R$ at $v$. Hence $P=\{3,4\}$, which is the medial of the tetrahedron.

The case when $R=\{5,3\}$ is more complicated. By arguments as above we find that $q=6$, and that the vertex-figure at a vertex $v$ of $P$ is a planar hexagon with $D_{3}$-symmetry and with vertices among those of $R$. It is easy to see that only two configurations for the convex hull of the vertex-figure at $v$ are possible, as indicated by the yellow and blue polygons in Figure 3. In the first (yellow) configuration for the convex hull, the vertex-figure of $P$ at $v$ has as its vertices the vertices of the three pentagons of $R$ at $v$ that lie on edges opposite to $v$ on these pentagons. In the second (blue) configuration for the convex hull, the vertices are the antipodes of the vertices of the hexagon in the first configuration. We next consider these two configurations in turn to show that the first leads to three hereditary polyhedra with non-central planar faces, and that the second cannot occur.

For the first configuration of the convex hull three scenarios are possible and each contributes one polyhedron.

First suppose that the vertex-figure of $P$ at $v$ is a convex hexagon and thus coincides with its convex hull. Then the edges opposite to $v$ on the pentagon faces of $R$ at $v$ are among the edges of the vertex-figure of $P$ at $v$. In $P$, these edges appear as the vertexfigures of pentagram faces $\left\{\frac{5}{2}\right\}$ at $v$ inscribed in the pentagon faces of $R$ at $v$. The other 


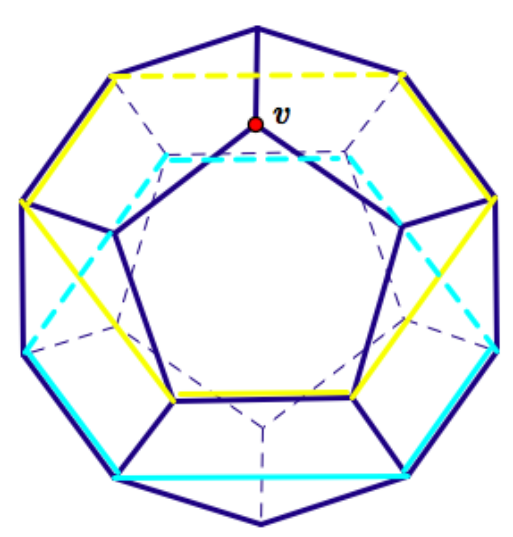

Figure 3: Configurations for the convex hull of the vertex-figure at $v$ when $P$ has the same vertex-set as $R=\{5,3\}$.

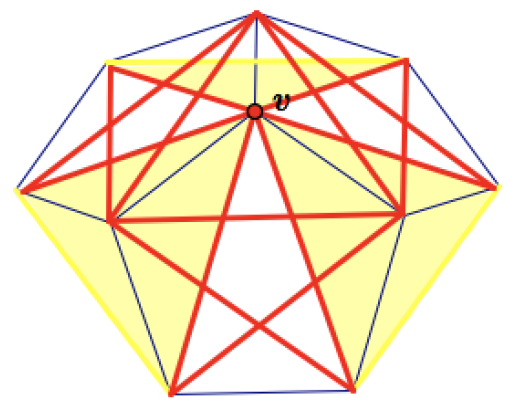

Figure 4: Faces of $P$ at $v$, for the yellow (first) configuration of Figure 3 when the vertexfigure at $v$ is a convex hexagon.

faces of $P$ at $v$ are equilateral triangles formed by the three vertices that are adjacent in $R$ to a neighbour of $v$ in $R$. Thus three pentagrams and three triangles alternate around $v$ in $P$, as illustrated in Figure 4. The resulting polyhedron $P$ is the uniform polyhedron $\left(\frac{5}{2} \cdot 3\right)^{3}$ called the small ditrigonal icosidodecahedron (see [2]).

Next suppose that (still in the first configuration for the convex hull) the vertex-figure of $P$ at $v$ is not a convex hexagon. In this case the vertex-figure is a non-convex hexagon of one of two kinds.

The first kind of non-convex hexagonal vertex-figure is indicated with dashed red lines in Figure 5. Here the edges opposite to $v$ on the pentagon faces of $R$ at $v$ are not among the edges of the vertex-figure of $P$ at $v$. The faces of $P$ at $v$ again are of two kinds alternating around $v$. There are three regular convex pentagons "cutting across" $R$ (shown in heavy red lines in Figure 5), and there are three equilateral triangles of the same kind as before, each formed by the three vertices that are adjacent in $R$ to a neighbour of $v$ in $R$. Now $P$ is the uniform polyhedron $(5 \cdot 3)^{3}$ called the great ditrigonal icosidodecahedron (see [2]). 


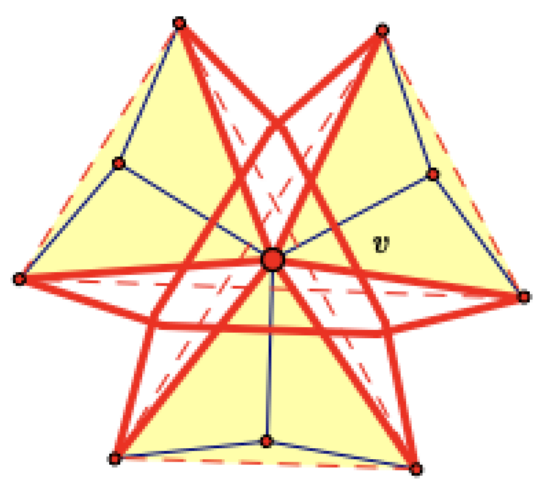

Figure 5: Faces of $P$ at $v$, for the yellow (first) configuration of Figure 3 when the vertexfigure at $v$ is a non-convex hexagon not sharing any edges with $R=\{5,3\}$.

The second kind of non-convex hexagonal vertex-figure is indicated with dashed red lines in Figure 6. Now the edges opposite to $v$ on the pentagon faces of $R$ at $v$ are edges of the vertex-figure of $P$ at $v$. Again two kinds of faces of $P$ alternate at $v$. There are three regular convex pentagons "cutting across" $R$ (shown in heavy red lines in Figure 6), and there are three pentagrams inscribed in the pentagon faces of $R$ at $v$. Thus $P$ is the uniform polyhedron $\left(5 \cdot \frac{5}{2}\right)^{3}$ called the ditrigonal dodecadodecahedron (see [2]).

The second (blue) configuration of Figure 3 for the convex hull of the vertex-figure of $P$ at $v$ can be ruled out as follows. As for the first configuration of Figure 3, the vertex-figure of $P$ at $v$ must either be a convex hexagon identical with the convex hull, or a non-convex hexagon sharing three edges with the convex hull. In either case, each edge of the vertexfigure of $P$ at $v$ which is an edge of the convex hull is necessarily the vertex-figure of a face of $P$ at $v$, and therefore must span, together with $v$, the plane of this face. As this plane contains only three vertices of $R$, the face itself could only be a (non-regular) triangle, so $P$ could not be regular-faced. Thus the second configuration of Figure 3 cannot occur.

This completes the enumeration of the polyhedra $P$ for Case 1 . We next investigate the second possibility for the placement of vertices of $P$ relative to $R$. Recall that $q$ is even.

Case 2: The vertices of $P$ lie at the edge midpoints of $R$.

In this case necessarily $q=4$ since now the vertices of $P$ have only $D_{2}$-symmetry. Thus the vertex-figures are congruent planar 4-gons with $D_{2}$-symmetry. As pairs of dual Platonic solids yield the same set of edge midpoints up to similarity, it suffices to consider only the regular polyhedra $R=\{3,3\},\{3,4\}$, and $\{5,3\}$.

The first possibility can be ruled out immediately. If $R=\{3,3\}$, then the (planar) faces of $P$ must be regular triangles since $R$ has only rotations of order 2 or 3 . The vertices of $P$ are just those of a regular octahedron, and the vertex-figures are given by equatorial squares of this octahedron. Hence $P$ must coincide with this octahedron. But then $G(P) \neq G(R)$, which contradicts our choice of $R$. (Recall that the octahedron occurred as the medial of $\{3,3\}$ in Case 1.) Thus this choice $R$ does not contribute a polyhedron.

If $R=\{3,4\}$, then the faces of $P$ must be regular triangles or squares since $R$ has only rotations of order 2,3 or 4 . Now the vertices of $P$ are just those of a cuboctahedron 


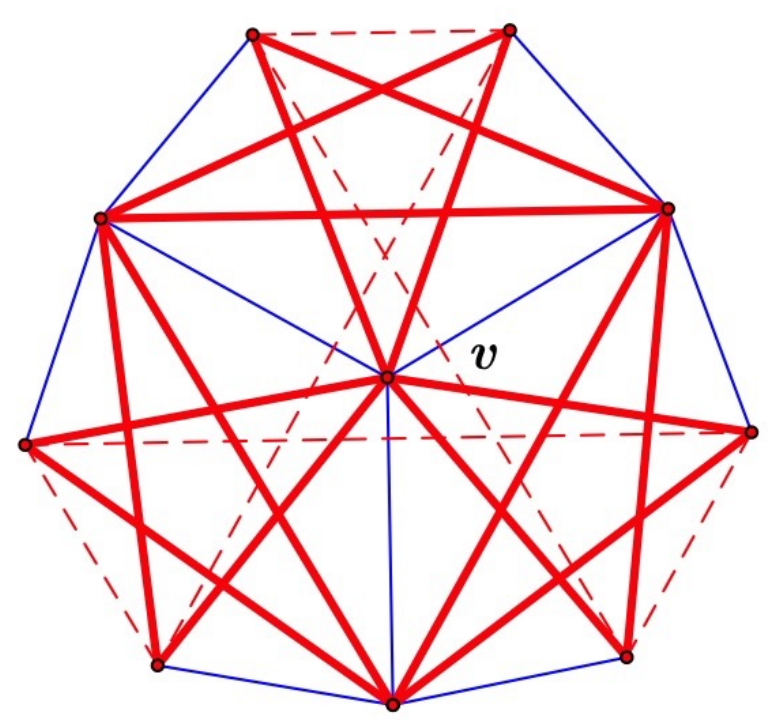

Figure 6: Faces of $P$ at $v$, for the yellow (first) configuration of Figure 3 when the vertexfigure at $v$ is a non-convex hexagon sharing edges with pentagonal faces of $R=\{5,3\}$ at $v$.

$\left\{\begin{array}{l}3 \\ 4\end{array}\right\}$. The vertex-figure at a vertex $v$ of $P$ must necessarily be convex. In fact, opposite vertices of the convex hull of the vertex-figure at $v$ cannot be joined (in a bowtie fashion) by an edge of the vertex-figure, since otherwise the planar face at $v$ determined by this edge would need to be central, in violation to our standing assumption in this section that $P$ has no central faces. There are only two possible configurations (shown in yellow and light blue in Figure 7) for the four neighbours of $v$ in $P$.

In the first (yellow) configuration, the neighbors of $v$ in $P$ are the same as those of $v$ in the cuboctahedron. In this case $P$ must coincide with the cuboctahedron and thus be the medial of $\{3,4\}$. In fact, the triangular faces of $P$ at $v$ must be just those of the cuboctahedron, and then this must also hold for the square faces.

In the second (light blue) configuration, the neighbors of $v$ in $P$ are the antipodal points of those in the first configuration. But this choice can be ruled out since the triangular faces at $v$ could not be regular.

If $R=\{3,5\}$, then the faces of $P$ must be regular triangles, convex pentagons, or pentagrams, since $R$ has only rotations of order 2, 3 or 5. Now the vertices of $P$ are just those of an icosidodecahedron $\left\{\begin{array}{l}3 \\ 5\end{array}\right\}$.

Any triangular face of $P$ must either be inscribed in a triangle face of $R$ as shown in Figure 8(a), or have as its vertices the midpoints of the edges of $R$ which emanate from the vertices of a triangle face of $R$ but do not belong to the adjacent triangle faces (see Figure 8(b)). Clearly, not all faces of $P$ can be triangles. Similarly, by the $D_{5}$-symmetry of the pentagonal faces of $P$, there can only be two possible configurations for the vertex sets of pentagonal faces of $P$. The convex hulls of these vertex sets are shown in Figures $8(\mathrm{c}, \mathrm{d})$. 


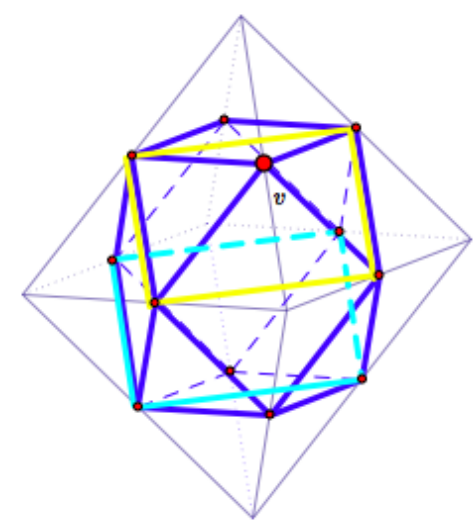

Figure 7: Possible convex hulls of the vertex-figures of $P$ at $v$ when the vertices of $P$ lie at the edge midpoints of an octahedron.

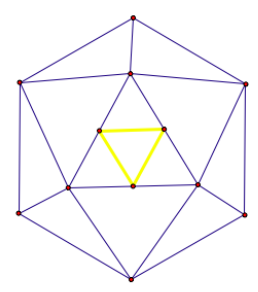

(a)

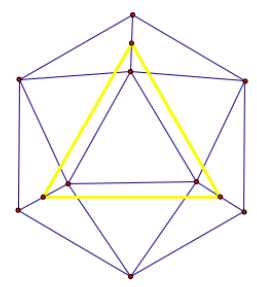

(b)

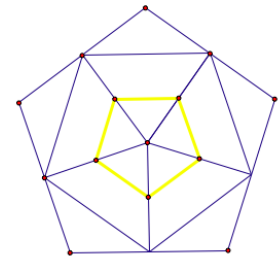

(c)

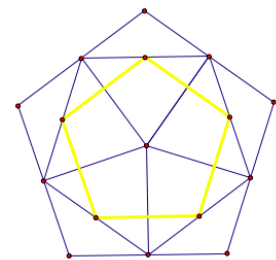

(d)

Figure 8: Possible convex hulls of the faces of $P$ when the vertices of $P$ lie at the edge midpoints of $\{3,5\}$. 
First suppose that $P$ indeed has a triangular face. If the triangular faces are positioned as in Figure 8(a), then the pentagonal faces must be convex and $P$ itself must be an icosidodecahedron, the medial of the icosahedron. On the other hand, if the triangular faces are as in Figure 8(b), then the pentagonal faces must be pentagrams with vertex sets located as in Figure 8(d), and $P$ itself must be $\left\{\begin{array}{l}3 \\ \frac{5}{2}\end{array}\right\}$, the medial of the Kepler-Poinsot polyhedron $\left\{3, \frac{5}{2}\right\}$ (or its dual $\left\{\frac{5}{2}, 3\right\}$ ).

If $P$ has no triangular faces, then all its faces must be convex pentagons or pentagrams. If a pentagonal face with vertices located as in Figure 8(c) occurs, then this must be a pentagram face whose adjacent faces are convex pentagon faces with vertex sets located as in Figure $8(\mathrm{~d})$. Then $P$ must be $\left\{\begin{array}{c}5 \\ \frac{5}{2}\end{array}\right\}$, the medial of $\left\{5, \frac{5}{2}\right\}$ (or its dual $\left\{\frac{5}{2}, 5\right\}$ ). Lastly, we can rule out the possibility that all pentagonal faces of $P$ have vertex sets as in Figure 8(d). In fact, otherwise all faces of $P$ must be convex pentagons, or all faces of $P$ must be pentagrams, in both cases with four faces meeting at a vertex. But the vertex configurations of Figure 8(d) arising from two different vertices of the icosahedron $R$ (each corresponding to a point like the central point in the figure) can never intersect in more than one point, so adjacent faces of $P$ cannot both be convex pentagons or pentagrams.

This settles the enumeration of the polyhedra $P$ for Case 2 , and completes the proof of Proposition 3.2.

\section{Planar-faced polyhedra with a central face}

In this section, we treat the finite hereditary polyhedra $P$ with planar regular faces some of which are central. So let $P$ be a polyhedron of this kind. Recall from Section 2 that then each edge of $P$ must lie in a central planar face and a non-central planar face, and that $G(P)$ must have two face orbits given by the central faces respectively the non-central faces of $P$. Moreover, the vertex valency $q$ is even and the vertex-figures are planar.

First observe that the reflections in the perpendicular bisectors of edges of $P$ are symmetries of $P$. In fact, the planar symmetry group of the non-central face at a given edge, trivially extended to a subgroup of $G(P)$, is generated by plane reflections and in particular contains the reflection in the perpendicular bisector of this edge.

Next we need to analyze the way in which the planar symmetries of central faces $F$ that interchange the two edges of $F$ at a vertex of $F$ appear in $G(P)$. It turns out that there can only be two possible scenarios: either all such symmetries appear as plane reflections, or all appear as half-turns. In particular we will see that the first scenario will not occur.

The goal of this section is to prove the following proposition.

Proposition 4.1. Let $P$ be a (finite) hereditary polyhedron with regular planar faces, including some central faces. Then $P$ is the medial of the Petrie dual of either a Platonic solid or a Kepler-Poinsot polyhedron.

Proof. The proof investigates the two possible ways (Cases 1 and 2 below, respectively) in which the planar reflective symmetries of a central face are extended to symmetries of $P$, namely either all as plane reflections or one half as plane reflections (interchanging the two vertices of an edge) and the other half as half-turns (interchanging the two edges at a vertex). The behavior is uniform across all central faces, since any two central faces are equivalent under $G(P)$. If the reflective symmetries of all central faces are extended to $P$ by plane reflections, then the reflective symmetries of all faces of $P$ are extended to $P$ by plane reflections, since we know this to be true for the non-central faces. 
Now suppose $P$ is a finite hereditary polyhedron with regular planar faces, including some central faces.

Case 1: The planar reflective symmetries of central faces are extended to $P$ by plane reflections.

We show that this case does not occur; in other words, there are no polyhedra with central faces in which all reflective symmetries of all faces are extended by plane reflections.

Suppose that $P$ is a polyhedron with central faces such that all planar reflective symmetries of all faces are extended to $P$ by plane reflections. Then all subgroups of $G(P)$ extending planar symmetry groups of faces of $P$ are generated by plane reflections. Again, as in Section 3, the vertices of $P$ must lie at the vertices or the edge midpoints of a Platonic solid $R$ with $G(R)=G(P)$.

The case $R=\{3,3\}$ can be eliminated as follows. In this case the vertices of $P$ could only be the six edge midpoints of $R$, since otherwise $P$ could not have a central face. Then the central faces of $P$ could only be given by the three equatorial squares of the octahedron formed by these six vertices, and the non-central faces by four alternate triangle faces of this octahedron. Thus $P$ would have to be $\operatorname{Me}\left(\{3,3\}^{\pi}\right)$, the medial of the Petrial of $\{3,3\}$. However, the planar reflective symmetries of the central faces of $\operatorname{Me}\left(\{3,3\}^{\pi}\right)$ that interchange the edges at a vertex are not extended to $\operatorname{Me}\left(\{3,3\}^{\pi}\right)$ by plane reflections, so in the present context this polyhedron must be rejected by our case assumption. Note, however, that $\operatorname{Me}\left(\{3,3\}^{\pi}\right)$ will occur as a legitimate polyhedron in Case 2 .

Now let $R=\{3,4\}$. If the vertices of $P$ are just those of $R$, then again $P$ must be the medial of the Petrial of a tetrahedron and can be eliminated as before (or here, alternatively, because $G(P) \neq G(R)$ ). The case when the vertices of $P$ are the edge midpoints of $R$ (that is, the vertices of a cuboctahedron) can be ruled out as follows. Since a central face would need to have full dihedral symmetry, it could only be a triangle or square. There are no central triangles with $D_{3}$-symmetry spanned by vertices of a cuboctahedron, so the central faces could only be squares. However, the squares inscribed as central squares in the vertex-set of a cuboctahedron are such that each vertex of the cuboctahedron can only lie in one such square. Thus this possibility is excluded as well.

The cube $R=\{4,3\}$ also does not contribute a polyhedron. The case of vertex placements for $P$ at the edge-midpoints of $R$ is the same as for $\{3,4\}$ and can again be ruled out. The vertices of $P$ also cannot lie at the vertices of $R$, since there are no central regular polygons spanned by vertices of the cube.

The two cases $R=\{3,5\}$ and $R=\{5,3\}$ similarly do not give a polyhedron. In fact, the central regular faces of $P$ would have to be triangles, pentagons or pentagrams. But no such faces can be placed with full dihedral symmetry. This applies to both kinds of vertex placements for $P$ on $R$.

In summary, Case 1 does not lead to a hereditary polyhedron of the desired kind.

Case 2: Some planar reflective symmetries of central faces are not extended to P by plane reflections.

We know from our previous discussion that the reflective symmetries of faces which are not extended to $P$ by plane reflections, are just the reflective symmetries of central faces which interchange the two edges at a vertex, and that these are extended to $P$ by half-turns. Thus the subgroups of $G(P)$ extending symmetry groups of central faces are generated by a plane reflection and a half-turn. In particular, the central faces must have an even number 
of vertices. The subgroups of $G(P)$ extending symmetry groups of non-central faces still are generated by two plane reflections.

We show that $q=4$ and that the vertices of $P$ must lie on axes of 2 -fold rotation. Suppose $v$ is a vertex, $F$ a central face at $v$, and $G$ a non-central face at $v$ adjacent to $F$. Let $r_{F}$ and $r_{G}$ respectively denote the extended symmetries of the faces $F$ and $G$ that interchange the edges at $v$. Then it is clear that the product $r_{F} r_{G}$ has order $q / 2$. (Recall that $q$ is even.) On the other hand, $r_{F}$ is the half-turn about the line through $o$ and $v$, and $r_{G}$ is a reflection in a plane through $o$ and $v$ perpendicular to the plane of $G$. Hence, since the rotation axes of $r_{F}$ lies in the reflection plane of $r_{G}$, the product $r_{F} r_{G}$ must be a reflection in the plane which is perpendicular to the reflection plane of $r_{G}$ and meets this plane in the rotation axis of $r_{F}$. Thus $q=4$, and there are just two central faces and two non-central faces meeting in alternating fashion at $v$. If $F^{\prime}$ and $G^{\prime}$ respectively are the central and noncentral faces of $P$ at $v$ distinct from $F$ and $G$, and $r_{F^{\prime}}$ and $r_{G^{\prime}}$ are the extended symmetries of $F^{\prime}$ and $G^{\prime}$ defined in the same way as $r_{F}$ and $r_{G}$ for $F$ and $G$, then necessarily $r_{F^{\prime}}=r_{F}$ and $r_{G^{\prime}}=r_{G}$, and $r_{F} r_{G}$ interchanges $F$ and $F^{\prime}$, and $G$ and $G^{\prime}$.

It follows as before that the vertices of $P$ must lie at the vertices or edge midpoints of a Platonic solid $R$ with $G(R)=G(P)$. Clearly, by what we just said, the vertices of $P$ could only lie at vertices of $R$ if $R=\{3,4\}$ or $\{4,3\}$ (but below these possibilities will be ruled out as well).

If $R=\{3,3\}$ then vertex placements for $P$ at the edge midpoints of $R$ are possible precisely for the reason that they were ruled out under Case 1 . In fact, the resulting polyhedron is $\operatorname{Me}\left(\{3,3\}^{\pi}\right)$, the medial of the Petrie dual of $\{3,3\}$, also known as the tetrahemihexahedron (see [2]). In $\operatorname{Me}\left(\{3,3\}^{\pi}\right)$, the planar symmetries of the central faces that interchange the edges at a vertex indeed are extended by half-turns, not plane reflections. Thus $R=\{3,3\}$ contributes $\operatorname{Me}\left(\{3,3\}^{\pi}\right)$.

Now let $R=\{3,4\}$. In this case the vertex placements for $P$ at the vertices of $R$ can be ruled out, since the only possible candidate for a polyhedron, $\operatorname{Me}\left(\{3,3\}^{\pi}\right)$, has a smaller symmetry group than $R$. This polyhedron occurred in the previous case for $R$. On the other hand, the vertex placements for $P$ at the edge midpoints of $R$ lead to two possible polyhedra, as we can see as follows. First note that, under the assumption of Case 2, the only possible central faces are the equatorial hexagons of the cuboctahedron determined by the edge midpoints of $R$, or triangles with vertices among those of an equatorial hexagon. The latter are excluded since the central faces must have an even number of vertices. Thus the central faces are the equatorial hexagons of the cuboctahedron. The non-central faces must necessarily be triangles or squares, as only these have dihedral symmetry. In either case the non-central faces must be faces of the cuboctahedron. If the non-central faces are triangles, then $P$ is the medial of the Petrie dual of a cube, $\operatorname{Me}\left(\{4,3\}^{\pi}\right)$, also called the octahemioctahedron [2]. If the non-central faces are squares, then $P$ is the medial of the Petrie dual of the octahedron, $\operatorname{Me}\left(\{3,4\}^{\pi}\right)$, also called the cubohemioctahedron [2].

For $R=\{4,3\}$, the polyhedron $P$ cannot have its vertices at the vertices of $R$, since a central face could not be regular. On the other hand, by duality, the vertex placements for $P$ at the edge-midpoints of $R$ result in the same two polyhedra as in the previous case.

Now let $R=\{3,5\}$. Suppose the vertices of $P$ lie at the edge midpoints of $R$. The central faces all must have 2-fold, 3-fold, or 5-fold rotational symmetry, as well as an even number of vertices, and thus must be squares, hexagons, or decagons. Squares can be ruled out immediately. In fact, although a square can be placed as a central square with its vertices at edge midpoints of $R$, this cannot be done in such a way that all symmetries of 


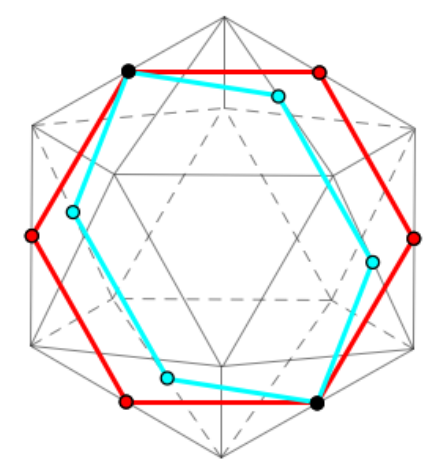

Figure 9: Central hexagonal faces with vertices at edge midpoints of an icosahedron.

the square extend to symmetries of $P$ (or equivalently, $R$ ), so $P$ could not be hereditary.

On the other hand, hexagonal central faces indeed can occur. Figure 9 shows how the vertices of a central regular hexagon can be placed at the edge midpoints of $R$, in such a way that the half-turns about the edges of $R$ that contain a vertex of this hexagon map the hexagon to itself. Each pair of antipodal vertices of this hexagon also lies in another central hexagon of the same kind. These two hexagons are interchanged by the reflection in the plane spanned by the pair of antipodal edges of $R$ determining the common vertices of the hexagons. Note that the six edges of $R$ whose midpoints are the vertices of any such hexagon form a regular skew hexagon centered at $o$; this is a 2-zigzag of $R$ (see [16, p. 196]). At each vertex $v$ of $P$, two central hexagonal faces and two non-central faces meet in an alternating fashion. The angle at $v$ between an edge of a central hexagon at $v$, and an edge of the other central hexagon at $v$, is either $2 \pi / 5$ or $3 \pi / 5$ (see again Figure 9 ). Thus, in between the two central hexagons meeting at $v$ can fit only two regular convex pentagons or two regular pentagrams. If the pentagonal faces are convex, then $P$ is $\operatorname{Me}\left(\left\{\frac{5}{2}, 5\right\}^{\pi}\right)$, the medial of the Petrie dual of the regular star polyhedron $\left\{\frac{5}{2}, 5\right\}$, with vertex-symbol $\left(5 \cdot 6^{*}\right)^{2}$, also called the great dodecahemi-icosahedron [2]. If the pentagonal faces are pentagrams, then $P$ is $\operatorname{Me}\left(\left\{5, \frac{5}{2}\right\}^{\pi}\right)$, the medial of the Petrie dual of the regular star polyhedron $\left\{5, \frac{5}{2}\right\}$, with vertex-symbol $\left(\frac{5}{2} \cdot 6^{*}\right)^{2}$, called the small dodecahemi-icosahedron [2].

There are also four hereditary polyhedra $P$ where the central faces are regular decagons (and the vertices still are at the edge midpoints of $R$ ). Their central faces are regular convex decagons $\{10\}$ or regular star decagons $\left\{\frac{10}{3}\right\}$, with each central face lying in a plane perpendicular to a 5-fold rotation axis of $R$.

If the central faces of $P$ are convex decagons, then each pair of antipodal vertices of a central decagon also lies in another central decagon of the same kind, as shown in Figure 10. This only leaves room at a vertex for non-central faces which are regular triangles or regular convex pentagons. If the non-central faces are triangles, then $P$ is $\operatorname{Me}\left(\{5,3\}^{\pi}\right)$, the medial of the Petrie dual of a dodecahedron, with vertex-symbol $\left(3 \cdot 10^{*}\right)^{2}$, known as the small icosihemidodecahedron [2]. If the non-central faces are convex pentagons, then $P$ is $\operatorname{Me}\left(\{3,5\}^{\pi}\right)$, the medial of the Petrie dual of an icosahedron, with vertex-symbol $\left(5 \cdot 10^{*}\right)^{2}$, called the small dodecahemidodecahedron [2]. 


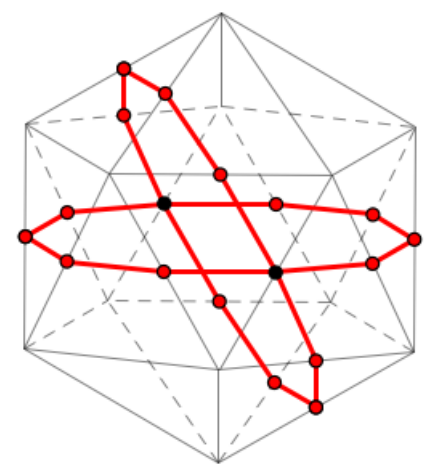

Figure 10: Central decagonal faces with vertices at edge midpoints of an icosahedron.

On the other hand, if the central faces of $P$ are star decagons $\left\{\frac{10}{3}\right\}$, which we may picture as inscribed in a regular decagon of the kind shown in Figure 10, then the noncentral faces must either be regular triangles or regular pentagrams $\left\{\frac{5}{2}\right\}$. If the non-central faces are triangles, then $P$ is a great icosihemidodecahedron [2], $\operatorname{Me}\left(\left\{\frac{5}{2}, 3\right\}^{\pi}\right)$, the medial of the Petrie dual of $\left\{\frac{5}{2}, 3\right\}$, with vertex-symbol $\left(3 \cdot\left(\frac{10}{3}\right)^{*}\right)^{2}$. If the non-central faces are pentagrams, then $P$ is a great dodecahemidodecahedron [2], $\operatorname{Me}\left(\left\{3, \frac{5}{2}\right\}^{\pi}\right)$, the medial of the Petrie dual of $\left\{3, \frac{5}{2}\right\}$, with vertex-symbol $\left(\frac{5}{2} \cdot\left(\frac{10}{3}\right)^{*}\right)^{2}$.

Finally, appealing to duality, for $R=\{5,3\}$ the vertex placements for $P$ at the edge midpoints of $R$ produce the same four polyhedra as in the previous case.

This settles the enumeration of the polyhedra in Case 2. Now the proof of Proposition 4.1 is complete.

The final step of the proof of Theorem 1.1 consists of drawing together Propositions 3.1 and 4.1. Propositions 3.1 describes the finite polyhedra with no central planar face, while Propositions 4.1 deals with the polyhedra that have central planar faces. This leads to the desired result.

\section{The enumeration}

As pointed out earlier, several polyhedra listed in Theorem 1.1 are counted more than once in the theorem. For example, each pair of dual finite regular polyhedra gives the same medial. The Platonic solids and Kepler-Poinsot polyhedra each have a geometric dual which is also regular, but their Petrie duals do not. The Petrie duals of course have combinatorial duals, but these are not realizable as regular geometric polyhedra in $\mathbb{E}^{3}$. Leaving aside the octahedron, which is already counted in the list of Platonic solids but also occurs as the medial of the tetrahedron, we therefore can obtain at most $4+9=13$ different medials (other than the octahedron) from regular polyhedra. This then leaves at most 25 possible polyhedra.

Inspection of the 25 polyhedra shows that these are indeed different, that is, mutually geometrically non-similar. The arguments are based on a comparison of the vertex-symbols as well as on the existence and nature of central faces (if any). 
In Table 1, we list the 25 polyhedra along with the refined vertex-symbols, symmetry groups, and relevant internal references. Recall that the superscript $\pi$ denotes the Petriedual, and that the superscript " $*$ " in a vertex-symbol means that the corresponding face type represents a central face of the polyhedron. For example, the medial of the Petrie dual of the Kepler-Poinsot polyhedron $\left\{\frac{5}{2}, 3\right\}$, denoted $\operatorname{Me}\left(\left\{\frac{5}{2}, 3\right\}^{\pi}\right)$, has vertex-symbol $\left(3 \cdot\left(\frac{10}{3}\right)^{*}\right)^{2}$, indicating that two regular triangles $\{3\}$ and two central regular star-decagons $\left\{\frac{10}{3}\right\}$ alternate at a vertex. A vertex-symbol only contains a superscript " $*$ " if the polyhedron has a central face. The next to last column lists the symmetry groups, with $[p, q]$ denoting the symmetry group of the Platonic solids $\{p, q\}$. The last column of the table gives the internal reference where the corresponding polyhedron is described or derived; for example, 4.1/C2 means "Proposition 4.1, Case 2 of its proof".

\section{ORCID iDs}

Egon Schulte (D) https://orcid.org/0000-0001-9725-3589

Asia Ivić Weiss (iD https://orcid.org/0000-0003-4937-2246

\section{References}

[1] H. S. M. Coxeter, Regular Polytopes, Dover Publications, New York, 3rd edition, 1973.

[2] H. S. M. Coxeter, M. S. Longuet-Higgins and J. C. P. Miller, Uniform polyhedra, Philos. Trans. Roy. Soc. London. Ser. A. 246 (1954), 401-450, doi:10.1098/rsta.1954.0003.

[3] H. S. M. Coxeter and W. O. J. Moser, Generators and Relations for Discrete Groups, volume 14 of Ergebnisse der Mathematik und ihrer Grenzgebiete, Springer-Verlag, Berlin, 4th edition, 1980.

[4] A. W. M. Dress, A combinatorial theory of Grünbaum's new regular polyhedra, Part I: Grünbaum's new regular polyhedra and their automorphism group, Aequationes Math. 23 (1981), 252-265, doi:10.1007/BF02188039.

[5] A. W. M. Dress, A combinatorial theory of Grünbaum's new regular polyhedra, part II: Complete enumeration, Aequationes Math. 29 (1985), 222-243, doi:10.1007/BF02189831.

[6] B. Grünbaum, Regular polyhedra—old and new, Aequationes Math. 16 (1977), 1-20, doi:10. 1007/BF01836414.

[7] B. Grünbaum, "New" uniform polyhedra, in: A. Bezdek (ed.), Discrete Geometry, Dekker, New York, volume 253 of Monographs and Textbooks in Pure and Applied Mathematics, pp. 331-350, 2003, doi:10.1201/9780203911211.ch23, in honor of W. Kuperberg's 60th birthday.

[8] I. Hubard, M. del Río Francos, A. Orbanić and T. Pisanski, Medial symmetry type graphs, Electron. J. Combin. 20 (2013), \#P29 (28 pages), https : / /www. combinatorics.org/ ojs/index.php/eljc/article/view/v20i3p29.

[9] I. Hubard, A. Orbanić and A. Ivić Weiss, Monodromy groups and self-invariance, Canad. J. Math. 61 (2009), 1300-1324, doi:10.4153/CJM-2009-061-5.

[10] N. W. Johnson, Uniform Polytopes, unpublished book manuscript.

[11] N. W. Johnson, Convex polyhedra with regular faces, Canadian J. Math. 18 (1966), 169-200, doi:10.4153/CJM-1966-021-8.

[12] H. Martini, A hierarchical classification of Euclidean polytopes with regularity properties, in: T. Bisztriczky, P. McMullen, R. Schneider and A. I. Weiss (eds.), Polytopes: Abstract, Convex and Computational, Kluwer Academic Publishers, Dordrecht, volume 440 of NATO Advanced Science Institutes Series C: Mathematical and Physical Sciences, 1994 pp. 71-96, doi: 
10.1007/978-94-011-0924-6_4, proceedings of the NATO Advanced Study Institute held in Scarborough, Ontario, August 20 - September 3, 1993.

[13] P. McMullen, Quasi-regular polytopes of full rank, in preparation.

[14] P. McMullen, Geometric Regular Polytopes, 2020, in press.

[15] P. McMullen and E. Schulte, Regular polytopes in ordinary space, Discrete Comput. Geom. 17 (1997), 449-478, doi:10.1007/PL00009304.

[16] P. McMullen and E. Schulte, Abstract Regular Polytopes, volume 92 of Encyclopedia of Mathematics and its Applications, Cambridge University Press, Cambridge, 2002, doi:10.1017/ CBO9780511546686.

[17] M. Mixer, E. Schulte and A. I. Weiss, Hereditary polytopes, in: R. Connelly, A. Ivić Weiss and W. Whiteley (eds.), Rigidity and Symmetry, Springer, New York, volume 70 of Fields Institute Communications, pp. 279-302, 2014, doi:10.1007/978-1-4939-0781-6_14.

[18] D. Pellicer and E. Schulte, Regular polygonal complexes in space, I, Trans. Amer. Math. Soc. 362 (2010), 6679-6714, doi:10.1090/S0002-9947-2010-05128-1.

[19] T. Pisanski and B. Servatius, Configurations from a graphical viewpoint, Birkhäuser Advanced Texts: Basler Lehrbücher. [Birkhäuser Advanced Texts: Basel Textbooks], Birkhäuser/Springer, New York, 2013, doi:10.1007/978-0-8176-8364-1.

[20] E. Schulte and A. I. Weiss, Skeletal geometric complexes and their symmetries, Math. Intelligencer 39 (2017), 5-16, doi:10.1007/s00283-016-9685-7.

[21] E. Schulte and A. Williams, Wythoffian skeletal polyhedra in ordinary space, I, Discrete Comput. Geom. 56 (2016), 657-692, doi:10.1007/s00454-016-9814-2.

[22] Wikipedia contributors, Wikipedia, The Free Encyclopedia, https://en.wikipedia. org/.

[23] A. Williams, Wythoffian skeletal polyhedra in ordinary space, II, in preparation.

[24] A. Williams, Wythoffian Skeletal Polyhedra, Ph.D. thesis, Northeastern University, Boston, Massachusetts, 2015, https://search.proquest.com/docview/1680014921.

[25] Wolfram Research, Inc., Mathematica, champaign, Illinois. 


\begin{tabular}{|c|c|c|c|c|}
\hline Polyhedra & Description & Vertex-symbol & Group & $\begin{array}{c}\text { Proposition/ } \\
\text { Case }\end{array}$ \\
\hline \multirow[t]{5}{*}{ Platonic } & $\{3,3\}$ & $(3)^{3}$ & {$[3,3]$} & 3.1 \\
\hline & $\{3,4\}=\operatorname{Me}(\{3,3\})$ & $(3)^{4}$ & {$[3,4]$} & $3.2 / \mathrm{C} 1$ \\
\hline & $\{4,3\}$ & $(4)^{3}$ & {$[3,4]$} & 3.1 \\
\hline & $\{3,5\}$ & $(3)^{5}$ & {$[3,5]$} & 3.1 \\
\hline & $\{5,3\}$ & $(5)^{3}$ & {$[3,5]$} & 3.1 \\
\hline \multirow[t]{4}{*}{ Kepler-Poinsot } & $\left\{3, \frac{5}{2}\right\}$ & $(3)^{5}$ & {$[3,5]$} & 3.1 \\
\hline & $\left\{\frac{5}{2}, 3\right\}$ & $\left(\frac{5}{2}\right)^{3}$ & {$[3,5]$} & 3.1 \\
\hline & $\left\{5, \frac{5}{2}\right\}$ & $(5)^{5}$ & {$[3,5]$} & 3.1 \\
\hline & $\left\{\frac{5}{2}, 5\right\}$ & $\left(\frac{5}{2}\right)^{5}$ & {$[3,5]$} & 3.1 \\
\hline \multirow[t]{13}{*}{ Medials } & $\operatorname{Me}(\{3,4\})=\operatorname{Me}(\{4,3\})$ & $(3 \cdot 4)^{2}$ & {$[3,4]$} & $3.2 / \mathrm{C} 2$ \\
\hline & $\operatorname{Me}(\{3,5\})=\operatorname{Me}(\{5,3\})$ & $(3 \cdot 5)^{2}$ & {$[3,5]$} & $3.2 / \mathrm{C} 2$ \\
\hline & $\operatorname{Me}\left(\left\{3, \frac{5}{2}\right\}\right)=\operatorname{Me}\left(\left\{\frac{5}{2}, 3\right\}\right)$ & $\left(3 \cdot \frac{5}{2}\right)^{2}$ & {$[3,5]$} & $3.2 / \mathrm{C} 2$ \\
\hline & $\operatorname{Me}\left(\left\{5, \frac{5}{2}\right\}\right)=\operatorname{Me}\left(\left\{\frac{5}{2}, 5\right\}\right)$ & $\left(5 \cdot \frac{5}{2}\right)^{2}$ & {$[3,5]$} & $3.2 / \mathrm{C} 2$ \\
\hline & $\operatorname{Me}\left(\{3,3\}^{\pi}\right)$ & $\left(3 \cdot 4^{*}\right)^{2}$ & {$[3,3]$} & $4.1 / \mathrm{C} 2$ \\
\hline & $\operatorname{Me}\left(\{3,4\}^{\pi}\right)$ & $\left(4 \cdot 6^{*}\right)^{2}$ & {$[3,4]$} & $4.1 / \mathrm{C} 2$ \\
\hline & $\operatorname{Me}\left(\{4,3\}^{\pi}\right)$ & $\left(3 \cdot 6^{*}\right)^{2}$ & {$[3,4]$} & $4.1 / \mathrm{C} 2$ \\
\hline & $\operatorname{Me}\left(\{3,5\}^{\pi}\right)$ & $\left(5 \cdot 10^{*}\right)^{2}$ & {$[3,5]$} & $4.1 / \mathrm{C} 2$ \\
\hline & $\operatorname{Me}\left(\{5,3\}^{\pi}\right)$ & $\left(3 \cdot 10^{*}\right)^{2}$ & {$[3,5]$} & $4.1 / \mathrm{C} 2$ \\
\hline & $\operatorname{Me}\left(\left\{3, \frac{5}{2}\right\}^{\pi}\right)$ & $\left(\frac{5}{2} \cdot\left(\frac{10}{3}\right)^{*}\right)^{2}$ & {$[3,5]$} & $4.1 / \mathrm{C} 2$ \\
\hline & $\operatorname{Me}\left(\left\{\frac{5}{2}, 3\right\}^{\pi}\right)$ & $\left(3 \cdot\left(\frac{10}{3}\right)^{*}\right)^{2}$ & {$[3,5]$} & $4.1 / \mathrm{C} 2$ \\
\hline & $\operatorname{Me}\left(\left\{5, \frac{5}{2}\right\}^{\pi}\right)$ & $\left(\frac{5}{2} \cdot 6^{*}\right)^{2}$ & {$[3,5]$} & $4.1 / \mathrm{C} 2$ \\
\hline & $\operatorname{Me}\left(\left\{\frac{5}{2}, 5\right\}^{\pi}\right)$ & $\left(5 \cdot 6^{*}\right)^{2}$ & {$[3,5]$} & $4.1 / \mathrm{C} 2$ \\
\hline \multirow[t]{3}{*}{ Exceptional } & & $(3 \cdot 5)^{2}$ & {$[3,5]$} & $3.2 / \mathrm{C} 1$ \\
\hline & & $\left(3 \cdot \frac{5}{2}\right)^{2}$ & {$[3,5]$} & $3.2 / \mathrm{C} 1$ \\
\hline & & $\left(5 \cdot \frac{5}{2}\right)^{2}$ & {$[3,5]$} & $3.2 / \mathrm{C} 1$ \\
\hline
\end{tabular}

Table 1: The 25 finite hereditary polyhedra with planar regular faces in $\mathbb{E}^{3}$. 